\title{
A systematic literature review of the management of chronic venous ulcers with autologous fibrin matrix with or without growth factors
}

\author{
Alejandra García Botero', Jorge Ernesto Cantini Ardila1', Luis David Devoz Borja1, Viviana Gómez-Ortega² \\ 'Plastic and Reconstructive Surgery Department, Hospital de San José, Fundación Universitaria de Ciencias de la Salud, Bogotá \\ 110111, Colombia. \\ ²Plastic and Reconstructive Surgery Department, Fundación Santa Fé de Bogotá, Bogotá 110111, Colombia.
}

Correspondence to: Dr. Alejandra García Botero, Servicio Cirugía Plástica Estética y Reconstructiva - Pabellón Arcadio Forero, Carrera 19 No. 8 - 32, Bogotá 110111, Colombia. E-mail: alebotero@hotmail.com

How to cite this article: García Botero A, Cantini Ardila JE, Devoz Borja LD, Gómez-Ortega V. A systematic literature review of the management of chronic venous ulcers with autologous fibrin matrix with or without growth factors. Plast Aesthet Res 2018;5:15. http://dx.doi.org/10.20517/2347-9264.2018.09

Received: 24 Feb 2018 First Decision: 21 Mar 2018 Revised: 3 Apr 2018 Accepted: 4 Apr 2018 Published: 23 Apr 2018

Science Editor: Raúl González-García Copy Editor: Guang-Zhe Zhu Production Editor: Cai-Hong Wang

\begin{abstract}
Aim: To evaluate available evidence for the effective management of venous leg ulcers with fibrin matrix with or without growth factors.
\end{abstract}

Methods: A systematic review of the literature was performed to evaluate the use of fibrin matrices with or without growth factors for the management of chronic venous ulcers in lower limbs. Article searches were performed in MEDLINE, EMBASE, COCHRANE, LILACS and ongoing clinical trials at ClinicalTrial.gov.

Results: The search in MEDLINE and EMBASE identified three articles; one was a pilot study evaluating the use of fibrin matrix and autologous growth factors that included patients with chronic ulcers of different etiology. The second article was a description of the product used in the previous study, and the third consisted of a series case reports of patient treated with cultured keratinocytes in a fibrin matrix. A COCHRANE searched resulted in one study assessing the cost effectiveness of using different fibrin matrices. The search in ClinicalTrial.gov and LILACS did not result in any findings.

Conclusion: The study did not provide a conclusive evidence for the use of fibrin matrices in patients with venous leg ulcers.

Keywords: Venous ulcers, legs, fibrin matrix, growth factors 


\section{INTRODUCTION}

Venous disease is a pathological chronic condition that causes significant morbidity and decreases quality of life in affected patients. The incidence of chronic venous insufficiency (CVI) varies within a range of $1 \%$ to $40 \%$ in women and $1 \%$ to $17 \%$ in men $^{[1]}$ with a higher prevalence in western industrialized countries. However, there is a low registration of cases due to the lack of reporting ${ }^{[2]}$. Patients with chronic peripheral vascular disease of venous origin exhibit secondary ulcer complications in skin and soft tissues of the lower limbs, with recurrence rates of $45 \%$ to $70 \%$ per year during the course of the patient's disease, which makes it difficult to manage ${ }^{[2]}$.

The pathophysiology of the venous ulcers is explained by the venous hypertension which lead to increased pressure in the distal veins of the leg; fibrin cuff theory in which the fibrin gets excessively deposited around capillary beds leading to elevated intravascular pressure causing enlargement of endothelial pores resulting in a further interstitial fibrinogen deposition increase. There is also an inflammatory trap theory in which various growth factors and inflammatory cells are trapped in the fibrin cuff promoting severe uncontrolled inflammation in surrounding tissue, thus preventing proper regeneration of wounds. Finally, the dysregulation of various pro-inflammatory cytokines and growth factors like tumor necrosis factoralpha, transforming growth factor (TGF) beta and matrix metalloproteinases lead to chronicity of the ulcers.

In order to determine the type of ulcer, it is very important to rule out arterial etiology. The clinical history may suggest the venous etiology. Additionally, the physical examination should describe the location, the measure of the size, the characteristics of the ulcer, the amount and type of exudate, the appearance of the ulcer bed, the odor of the ulcer and the pain associated with the ulcer.

Ankle brachial pressure index (ABPI) is a noninvasive test to determine the origin of the ulcer, which is evidence level B. This test uses the handheld doppler ultrasound, which identifies peripheral arterial disease in the leg. Systolic BP is measured at the brachial artery and at the ankle level.

$\mathrm{ABPI}=$ highest systolic foot pressure (dorsalis pedis/posterior tibial artery)/highest systolic brachial BP a. ABPI 0.8-1.2: indicative of good arterial flow. Suggestive of venous etiology if an ulcer is present

b. ABPI < 0.8: with the clinical picture of arterial disease-arterial insufficiency

c. ABPI > 1.2: suggestive of possible arterial calcification

A meta-analysis study of venous ulcers in an adult population reported a prevalence of $0.12 \%$ to $1.1 \%^{[1]}$. In the USA, seven million people suffer from CVI, which may be the underlying cause of $70 \%$ to $90 \%$ of ulcers in lower limbs ${ }^{[1]}$. The overall incidence of venous ulcers is considered to increase with age. In this regard, Evans et al..$^{[3]}$ reported a prevalence of venous ulcers of $56 \%$ in patients from 55 to 64 years of age, in comparison to $12 \%$ in patients from 18 to 24 years old.

Venous ulcers in lower limbs are one of the 10 most common medical problems in western countries with a substantial socioeconomic impact due to frequent disabilities ${ }^{[2]}$. In 1992, The American Venous Forum estimated that, in the USA at any point in time, one person/1000 has an unhealed venous ulcer ${ }^{[4]}$ that becomes a disability factor in multiple aspects of daily life, including the number of work days on the job. Ulcers in lower limbs not only affects older people but it also affects actively working people ${ }^{[5]}$, resulting in two million working days lost in USA ${ }^{[6]}$. Ulcers also diminish the quality of life since they can cause health consequences to the patient ${ }^{[3]}$ and to the social security health system as well. The treatment cost to patients with chronic venous ulcers in the USA is about three billion dollars per year, therefore it becomes a significant public health problem ${ }^{[7,8]}$. The elevated costs for treating this pathology has resulted in the development of new treatments with the objectives of reducing healing time, morbidity and associated costs. 
Current treatment of venous ulcers involves the application of compression therapy, with bandages or hosiery, along with different dressing types applied beneath the compression bandage or hosiery to enhance healing, create a humid environment and control exudates. However, several studies have reported non-significant differences when applying dressing types regarding time of healing and numbers of healed ulcers ${ }^{[2]}$.

The advancement of new biotechnologies has focused on the development of alternative therapies such as growing tissue in vitro, production of recombinant growth factors and tissue engineering. The use of autologous-derived products from the patient's blood, along with collagen or fibrin matrices, with or without cultured cells and autologous growth factors, has been suggested as an alternative therapy for treatment of chronic ulcers ${ }^{[9]}$. In vitro studies with animal models have reported a beneficial effect of growth factors, specifically platelet-derived growth factor (PDGF), fibroblast growth factor and granulocyte-macrophage colony-stimulating factor, on the proportion of healed ulcers ${ }^{[10-12]}$.

Fibrin matrices are a cost-effective option for ulcer management. Their source is from the blood of the same patient and they provide scaffolding for tissue growth, migration and cell regeneration. The beneficial effects of the fibrin matrix may be enhanced when it is used in conjunction with growth factors that stimulates cell proliferation.

The objective of the present review is to evaluate the available evidence for effective management of venous leg ulcers with autologous fibrin matrix with or without growth factors.

\section{METHODS}

We did a systematic review of studies evaluating the use of fibrin matrices with or without growth factors for the management of chronic venous ulcers in lower limbs. All studies were included without date restrictions. Articles searched in MEDLINE and EMBASE databases were performed in English. Available systematic reviews were searched in COCHRANE, and preliminary results and ongoing clinical trials were searched at ClinicalTrial.gov. The MESH terms corresponding to "fibrin" and "matrix", and "venous ulcer" were used in the search. The article searched was restricted to human reports. The LILACS database was used for searches in Spanish and Portuguese languages. Intervention was defined as the application of any autologous fibrin matrix (from the same patient with or without growth factors, for the treatment of venous ulcers in lower limbs).

\section{Criteria for inclusion}

The criteria for inclusion were studies evaluating patients with peripheral vascular disease of venous origin, who exhibited venous ulcers in lower limbs and received treatment with fibrin matrices with or without growth factors. Patients with chronic venous ulcers unhealed after 8 weeks of standard medical treatment.

\section{Criteria for exclusion}

Studies involving patients with ulcers of arterial origin or "mixed etiology" (defined as: ulcers from a combination of arterial and venous origin, venous insufficiency in pregnant women), patients with chronic osteomyelitis, diabetes with ulcers in the lower limbs, Marjolin's ulcers (ulcerating squamous cell carcinoma), malignant or terminal disease with an incidence of $\geq 5$ years, thermic, electric or radiation burns on the ulcerated area, vasculitis, chronic liver diseases, autoimmune diseases treated with immunosuppressant's, chemotherapy or radiotherapy, and diseases that affect wound healing, such as kidney insufficiency (patients in dialysis or receiving therapy following a kidney transplant), were excluded.

Also, studies of patients with concomitant use of others substances or products different than those evaluated in the present review, and those focused on compression instead of dressing therapy for the treatment of venous ulcers ${ }^{[13]}$ were excluded from the present literature review. 
The risk of bias was examined with the SIGN data analysis strategy, which assesses the internal validity and the quality assurance for each clinical study. A descriptive analysis was performed on the effective granulation in patients treated with fibrin matrix and with and without growth factors. The incidence of effective granulation was defined as those ulcers that healed completely or formed granulation tissue on $\geq 75 \%$ of the initial ulcer size. The mean time to healing or formation of granulation tissue was analyzed by using the Kaplan-Meier estimator. Absolute and relative frequencies were used to analyze the numbers of fibrin matrix applications required for effective granulation and its secondary consequences.

\section{RESULTS}

The literature searched in MEDLINE by using the MESH term, restricted to human reports and without restrictions to dates or types of study, identified 14 articles [Table 1]. Of these, five were selected by title, which fulfilled the objective of the present study, but only three were relevant to our study ${ }^{[9,14,15]}$. Analysis of abstracts from 12 articles, showed that management of venous ulcers were performed with different products, such as platelet-derived, platelet-enriched plasma or non-fibrin matrices. Since those 12 articles did not focus in fibrin matrix with autologous growth factors, they were excluded from the review.

From the three relevant articles found in MEDLINE ${ }^{[9,14,15]}$, the O'Connell et al. ${ }^{[9]}$ study was a pilot study, assessing the use of fibrin matrix and autologous growth factors for a period of 16 weeks, in 21 patients with chronic ulcers on lower limbs of different etiologies, including venous, arterial or a combination of both. Patients with ulcers of diabetic origin were included also, which was one of our exclusion criteria. In their pilot study, $66.7 \%$ of patients with venous ulcers showed completed ulcer healing within 7.1 weeks (median $=6$ weeks). The second article, from the same group as the pilot study, was a description of the Cascade ${ }^{\bullet}$ product that they used in the original study ${ }^{[14]}$. The third article, from Hartmann et al. ${ }^{[15]}$, primarily assessed a series of cases of seven patients with chronic venous ulcers treated with cultured keratinocytes transplanted in fibrin matrix. Results showed complete ulcer healing in 4 of 7 ulcers, with a mean healing time of 14.5 weeks; however, it was not possible to conclude that completed ulcer healing was a consequence of the presence of fibrin matrix or cultured keratinocytes.

The search in EMBASE database identified 35 articles, from which 4 were selected by title [Table 1]. The remaining 31 articles did not meet the search criteria and did not evaluate the intervention objective of the present systematic literature review. From 4 of the articles identified by their title, 3 were relevant and were the same articles found in the MEDLINE database search ${ }^{[9,14,15]}$. The search in COCHRANE (Central Register of Controlled Trials) identified 1 review of 3 randomized controlled trials assessing the cost effectiveness of using different fibrin matrices, such as bovine collagen matrix with neonatal keratinocytes, acellular matrix and poly-n-acetyl glucosamine matrices, on venous ulcers in lower limbs ${ }^{[16]}$.

The search of protocols and ongoing clinical trials at ClinicalTrial.gov database by using the MESH terms did find any reports [Table 2]. Similarly, the search in Spanish and Portuguese at the LILACS database did not find studies reported in either of the two languages [Table 1].

None of the four relevant articles selected by their summary met the criteria for inclusion as described in the material and methods section, and did not evaluate the intervention objective projected for our study [Table 3]; therefore, these articles were excluded from the review.

\section{DISCUSSION}

The primary treatment for venous ulcers involves application of compression therapy using bandages or compression hosiery ${ }^{[13]}$. In addition to compression therapy, different dressing types are applied beneath the compression bandage or hosiery, to enhance ulcer healing by creating a humid environment and controlling 
Table 1. Results of literature search in medical databases

\begin{tabular}{|c|c|c|c|c|c|c|}
\hline Database & Search terms & Search results & $\begin{array}{l}\text { Articles } \\
\text { selected }\end{array}$ & $\begin{array}{l}\text { Abstracts } \\
\text { selected }\end{array}$ & $\begin{array}{l}\text { Downloaded } \\
\text { articles }\end{array}$ & $\begin{array}{l}\text { Articles included } \\
\text { in the review }\end{array}$ \\
\hline MEDLINE & $\begin{array}{l}\text { Fibrin AND matrix AND } \\
\text { venous ulcers AND } \\
\text { venous leg ulcers }\end{array}$ & 14 & 5 & 3 & 3 & 0 \\
\hline EMBASE & $\begin{array}{l}\text { Fibrin AND matrix AND } \\
\text { venous ulcers AND } \\
\text { venous leg ulcers }\end{array}$ & 35 & 4 & 3 & 3 & 0 \\
\hline Cochrane & $\begin{array}{l}\text { Fibrin AND matrix AND } \\
\text { venous ulcers AND } \\
\text { venous leg ulcers }\end{array}$ & 1 & 1 & 1 & 1 & 0 \\
\hline LILACS & $\begin{array}{l}\text { Matrix de fibrina } Y \\
\text { ulcera venosa }\end{array}$ & 0 & 0 & 0 & 0 & 0 \\
\hline
\end{tabular}

General results from search in MEDLINE, EMBASE, Cochrane and LILACS databases by using search terms. The total numbers of search results, articles selected by the title associated with the objective of the present review, articles selected by summary and downloaded, and articles included in the review after evaluating the inclusion and exclusion criteria

Table 2. Search results of protocols and ongoing trials in ClinicalTrials.gov

\begin{tabular}{lccc}
\hline Search terms & Search results & Titles selected & $\begin{array}{c}\text { Protocols selected } \\
\text { Protocols included in the } \\
\text { review }\end{array}$ \\
\hline Fibrin AND matrix & 0 & 0 & 0 \\
AND & & & 0 \\
venous ulcer & & & \\
\hline
\end{tabular}

The search of protocols and ongoing clinical trials at ClinicalTrial.gov database did not show any results

Table 3. Criteria for exclusion of relevant articles selected by abstract and excluded from the review

\begin{tabular}{|c|c|}
\hline Relevant articles & Exclusion criteria \\
\hline $\mathrm{O}^{\prime}$ Connell et al. ${ }^{[9]}$ & $\begin{array}{l}\text { Included } 21 \text { patients with chronic ulcers in lower limbs of differing etiology: venous, arterial or a combination } \\
\text { of both }\end{array}$ \\
\hline O'Connell et al. ${ }^{[14]}$ & A description of the fibrin matrix product used in the article listed above \\
\hline Hartmann et al..$^{[15]}$ & Included a second intervention of cultured keratinocytes in a fibrin matrix \\
\hline Hankin et al. ${ }^{[16]}$ & $\begin{array}{l}\text { Included bovine collagen matrix with neonatal keratinocytes, acellular matrix and poly-n-acetyl glucosamine } \\
\text { matrices }\end{array}$ \\
\hline
\end{tabular}

Four relevant articles selected by summary during the search in MEDLINE and EMBASE were excluded from the review. (1) O'Connell et al. ${ }^{[9]}$ is a pilot study and not a clinical trial; (2) O'Connell et al. ${ }^{[14]}$ described a commercial product Cascade ${ }^{\circledR}$, but it is not a clinical trial; (3) Hartmann et $a /{ }^{[15]}$ is a series of case reports of seven patients and did not conclude if the ulcer healing was a consequence of the presence of fibrin matrix or cultured keratinocytes; and (4) Hankin et al ${ }^{[16]}$ is an analysis of the cost effectiveness of using different products as compared to that of fibrin matrix

exudates. However, several studies have reported no significant differences when applying dressing types regarding time of healing and numbers of healed ulcers ${ }^{[2]}$.

A systematic review of randomized controlled trials assessing the effectiveness of wound dressings indicated that certain dressing types used for the management of chronic venous ulcers, could in fact, not only enhance the rate of ulcers cured but also their healing time ${ }^{[17]}$. In contrast, a meta-analysis study of dressing types for venous ulcers published in 2011, reported non-significant differences in the numbers of ulcers cured or the healing rate between different dressing types ${ }^{[2]}$.

Wound dressings can be divided into non-occlusive or occlusive types and the latter further subdivided into three subcategories: semi-occlusive/occlusive, growth factors and human skin equivalents ${ }^{[17]}$. The function of non-occlusive and semi-occlusive dressings is prevention of loss of water vapor from the wound and acting as a thermal insulator, which are factors that promote the incidence and time of wound healing ${ }^{[18]}$. The growth factors dressings directly provide a specific growth factor to the wound, or indirectly enhance 
cellular growth and release of important substances for wound healing, while the human skin equivalents cover the wound and may also provide growth factors ${ }^{[17]}$.

Hydrocolloid dressings formed part of the occlusive dressing types and are usually composed of a matrix of sodium carboxymethyl cellulose with an adhesive elastomeric substance attached to a polymer base ${ }^{[19]}$. The hydrocolloid matrix absorbs exudates away from the wound surface ensuring a humid environment and promoting wound healing ${ }^{[20]}$.

In a systematic literature review of 20 randomized controlled trials evaluating whether complex wound dressings enhanced healing of venous ulcers, the author's reported that only $25 \%$ of the trials, and less than $10 \%$ of the overall studies, showed a significant proportion of healed ulcers by using these complex wound dressings ${ }^{[17]}$. Also, the use of hydrocolloid dressings did not enhance the proportion of ulcers healed in comparison to that of other dressing types, including growth factors. Similarly, a meta-analysis study from COCHRANE identified that hydrocolloid is the most evaluated dressing type, and an analysis of 27 (60\%) studies, indicated that there was no evidence that the use of hydrocolloid dressings for the treatment of chronic venous ulcers was more effective than other dressing types, and concluded that ulcer healing rates were not affected by the type of dressing used beneath compression ${ }^{[21]}$. Even though there was not enough data for most of dressing types to provide significant evidence of which type was more effective for healing venous ulcers, hydrocolloid dressings were more effective than low adherence dressings ${ }^{[21]}$. Nonetheless, none of these studies included growth factors dressing types or products with fibrin matrices, platelet-enrichedplasma or autologous growth factors in their analysis.

Regardless of the availability of new complex dressing types, the gauze is the dressing that is still most frequently used worldwide. Gauze is economical, easily available, absorbent and well known by health personal. Petrolatum or Vaseline impregnated gauzes commonly are used to provide a moist environment, and avoid desiccation that is conducive to wound healing ${ }^{[22]}$.

The advancement of new biotechnologies has focused on development of alternative therapies by growing tissue in vitro, producing recombinant growth factors and tissue engineering. In vitro studies with animal models have reported a beneficial effect of growth factors, specifically, PDGF, fibroblast growth factor and granulocyte-macrophage colony-stimulating factor, on the proportion of healed ulcers ${ }^{[9,1]}$. The practice of using growth factors for the treatment of chronic ulcers was the consequence of research results that demonstrated a significant reduction of localized growth factors in chronic wounds, resulting in cell cycle arrest and senescence of the wound bedding cells ${ }^{[10]}$.

Fibrin matrix and autologous growth factors became a cost-effective option for the management of patients with venous ulcers in the lower limbs. These biological products can be obtained from the same patient's blood, and provides scaffolding for tissue growth, migration and cell regeneration. The beneficial effects of the fibrin matrix may be enhanced when it is used with growth factors that stimulate cell proliferation. Currently, the challenge is producing an improved system for releasing high concentrations of growth factors to the bedding wound and establishing a close relationship between the bedding wound and Diana cells.

In our literature search we found little evidence for the efficacy of treating chronic venous ulcers with products containing fibrin matrix, platelet-enriched- plasma and autologous growth factors. In fact, we found only one pilot study that included patients with venous ulcers, but it also included patients with arterial ulcers and from other etiologies. The 12 patients with venous ulcers were treated with a product containing fibrin matrix, platelet-enriched- plasma and autologous growth factors, of which 8 (66.7\%; 64.7\% of the treated ulcers) showed completed ulcer healing within 7.1 weeks (median $=6$ weeks) after a mean of 
two applications per patient. The incidence of effective granulation $\geq 75 \%$ of the ulcer size was observed in $76.5 \%$ of the patients at 5 weeks of treatment initiation ${ }^{[9]}$. Also, we found a report case of seven patients with chronic venous ulcers treated with cultured keratinocytes which were transplanted in a fibrin matrix. Results showed complete ulcer healing in four of seven ulcers treated, with a mean healing time of 14.5 weeks ${ }^{[15]}$. However, it was not possible to conclude that completed ulcer healing was a consequence of the presence of fibrin matrix or cultured keratinocytes. The authors suggest, based on in vitro and in vivo studies, that the fibrin residue in cultured keratinocytes may inhibit complete wound healing after the transplantation. Therefore, the use of low density fibrin, instead of high density, to enhance grafting, keratinocytes survival and epithelialization was recommended.

\section{Study limitations}

The major limitation of the present study is the few studies available evaluating the management of venous ulcer with fibrin matrix and with or without growth factors. Most studies evaluated ulcers from different etiologies and ulcer management was done with a combination of fibrin matrix with different products or non-fibrin matrices. Therefore, it was not possible to perform a meaningful analysis of this type of intervention.

\section{Conclusions}

This review has not identified conclusive evidence for the use of fibrin matrices with or without growth factors in patients with venous leg ulcers. We found only a few studies that evaluated the results of this intervention.

\section{DECLARATIONS}

\section{Authors' contributions}

Study conception and design, critical revision: García Botero A

Acquisition of data, critical revision: Cantini Ardila JE

Analysis and Interpretation of data, critical revision: Devoz Borja LD

Drafting of manuscript, critical revision: Gómez-Ortega $\mathrm{V}$

\section{Data source and availability}

Article searches were performed in MEDLINE, EMBASE, COCHRANE, LILACS and ongoing clinical trials at ClinicalTrial.gov.

\section{Financial support and sponsorship}

None.

\section{Conflicts of interest}

There are no conflicts of interest.

\section{Patient consent}

Not applicable.

\section{Ethics approval}

Not applicable.

\section{Copyright}

(c) The Author(s) 2018. 


\section{REFERENCES}

1. Capeheart JK. Chronic venous insufficiency: a focus on prevention of venous ulceration. $J$ Wound Ostomy Continence Nurs 1996;23:227-34.

2. Abbade LP, Lastória S, Rollo HeA. Venous ulcer: clinical characteristics and risk factors. Int J Dermatol 2011;50:405-11.

3. González-Consuegra RV, Verdú J. Quality of life in people with venous leg ulcers: an integrative review. J Adv Nurs 2011;67:926-44.

4. Hume M. Venous ulcers, the vascular surgeon, and the Medicare budget. J Vasc Surg 1992;16:671-3.

5. Margolis DJ, Bilker W, Santanna J, Baumgarten M. Venous leg ulcer: incidence and prevalence in the elderly. J Am Acad Dermatol 2002;46:381-6.

6. Phillips T, Stanton B, Provan A, Lew R. A study of the impact of leg ulcers on quality of life: financial, social, and psychologic implications. J Am Acad Dermatol 1994;31:49-53.

7. Ruckley CV. Socioeconomic impact of chronic venous insufficiency and leg ulcers. Angiology 1997;48:67-9.

8. Van den Oever R, Hepp B, Debbaut B, Simon I. Socio-economic impact of chronic venous insufficiency. An underestimated public health problem. Int Angiol 1998;17:161-7.

9. O’Connell SM, Impeduglia T, Hessler K, Wang XJ, Carroll RJ, Dardik H. Autologous platelet-rich fibrin matrix as cell therapy in the healing of chronic lower-extremity ulcers. Wound Repair Regen 2008;16:749-56.

10. Knighton DR, Ciresi KF, Fiegel VD, Austin LL, Butler EL. Classification and treatment of chronic nonhealing wounds. Successful treatment with autologous platelet-derived wound healing factors (PDWHF). Ann Surg 1986;204:322-30.

11. Knighton DR, Ciresi K, Fiegel VD, Schumerth S, Butler E, Cerra F. Stimulation of repair in chronic, nonhealing, cutaneous ulcers using platelet-derived wound healing formula. Surg Gynecol Obstet 1990;170:56-60.

12. Celotti F, Colciago A, Negri-Cesi P, Pravettoni A, Zaninetti R, Sacchi MC. Effect of platelet-rich plasma on migration and proliferation of SaOS-2 osteoblasts: role of platelet-derived growth factor and transforming growth factor-beta. Wound Repair Regen 2006;14:195202.

13. Cullum N, Nelson EA, Fletcher AW, Sheldon TA. Compression bandages and stockings for venous leg ulcers. Cochrane Database Syst Rev 2000;(2):CD000265.

14. O'Connell SM, Hessler K, Dardik H. Cascade ${ }^{\circledR}$ Autologous system platelet-rich fibrin matrix in the treatment of chronic leg ulcers. $A d v$ Wound Care 2012;1:52-5.

15. Hartmann A, Quist J, Hamm H, Brocker EB, Friedl P. Transplantation of autologous keratinocyte suspension in fibrin matrix to chronic venous leg ulcers: improved long-term healing after removal of the fibrin carrier. Dermatol Surg 2008;34:922-9.

16. Hankin CS, Knispel J, Lopes M, Bronstone A, Maus E. Clinical and cost efficacy of advanced wound care matrices for venous ulcers. $J$ Manag Care Pharmacy 2012;18:375-84.

17. O'Donnell TF, Lau J. A systematic review of randomized controlled trials of wound dressings for chronic venous ulcer. $J$ Vasc Surg 2006;44:1118-25.

18. Winter GD. Formation of the scab and the rate of epithelization of superficial wounds in the skin of the young domestic pig. Nature 1962;193:293-4.

19. Cullum N. Leg ulcer treatments: a critical review (Part 2). Nurs Stand 1994;9:32-6.

20. Smith BA. The dressing makes the difference. Trial of two modern dressings on venous ulcers. Prof Nurse 1994;9:348, 350-2.

21. Palfreyman SJ, Nelson EA, Lochiel R, Michaels JA. Dressings for healing venous leg ulcers. Cochrane Database Syst Rev 2006;(3):CD001103.

22. Morin RJ, Tomaselli NL. Interactive dressings and topical agents. Clin Plast Surg 2007;34:643-58. 